نوري : تأثيرنوعية السوائل على أخواص الهندسية لصخور الحجر الجيزي من منطقة أسكي موصل

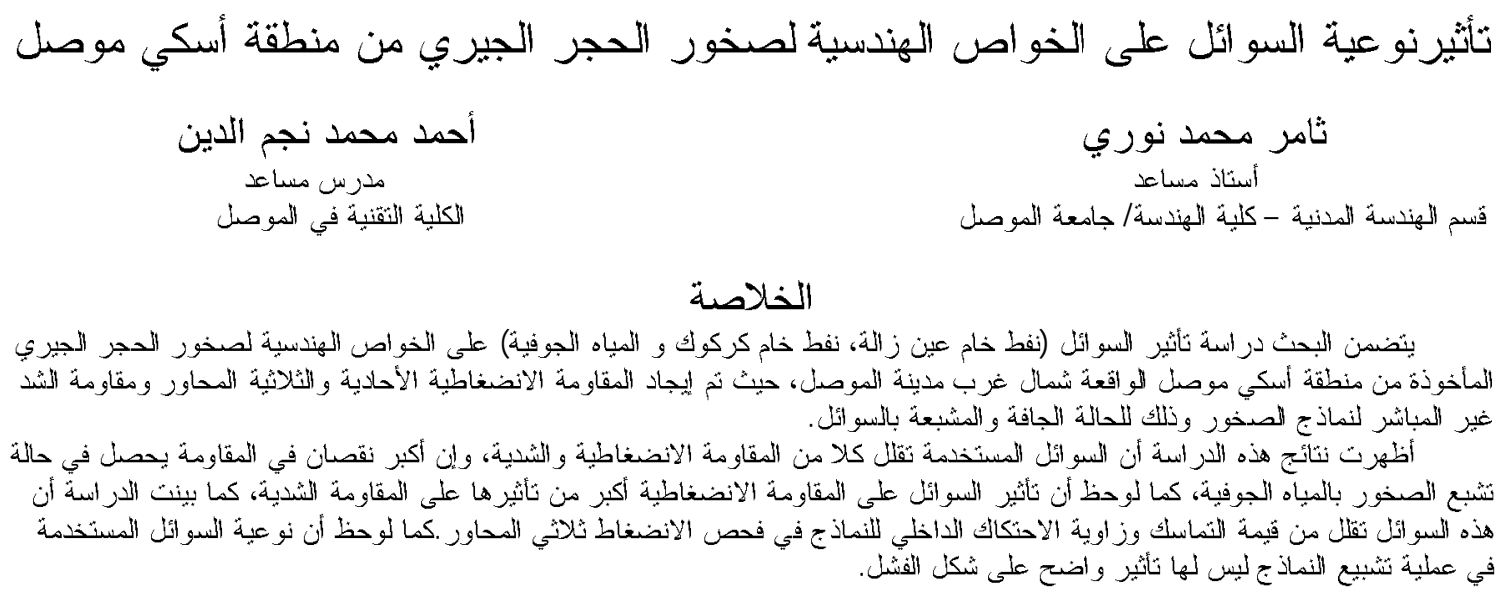

\title{
Effect of Liquids type on some Engineering Properties of Limestone Rock from Eski-Mosul
}

Thamer M. Nuri

Assis.Prof.

Civil Dept. Engg.College-M osul University

\author{
Ahmed M. Nejm Al-Deen \\ Assis. Lecturer \\ Technical College-Mosul
}

\begin{abstract}
This research studied the effect of liquids on the Engineering properties of the Limestone rocks. The liquids used were: crude oil from Ain Zala, crude oil from Kirkuk, natural ground Water.

The Limestone used was brought from Eski M osul situated at $45 \mathrm{Km}$ north west of M osul. Both the compressive and tensile tests were conducted on the Limestone rock specimens in the dry and saturated case using; Uniaxial, Triaxial Compression and Bending tests.

The tests showed that saturation with any one of the liquids lead to a decrease of both compressive and tensile strength. The greatest decrease occurred when ground water was used to saturate the specimens, and it was also noted that the effect of saturation gave greater percentage of decrease on the compressive than the tensile strength.

The study also showed that liquids lead to decrease in cohesion ( $\mathrm{c}$ ) and the Friction angle (Ф) obtained from triaxial test. The liquids used for saturation didn't have clear effect on the type and mode of failure on all specimens tested .

Keyword: $\sigma c:$ Compressive strength ot: Tensile strength
\end{abstract}




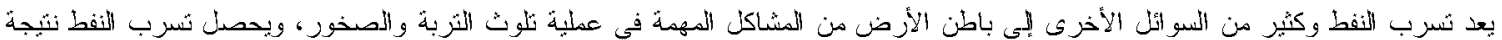

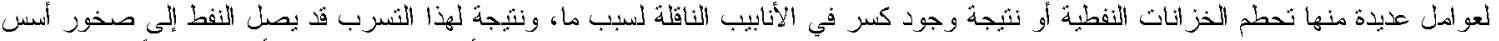

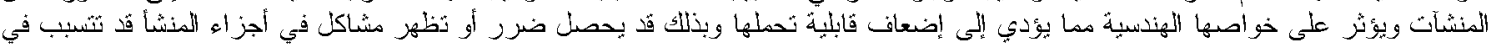

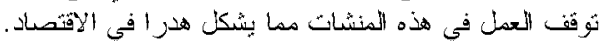

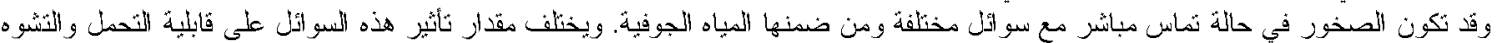

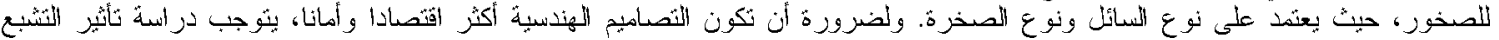

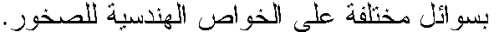

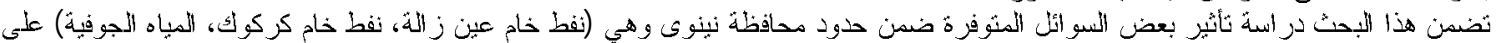

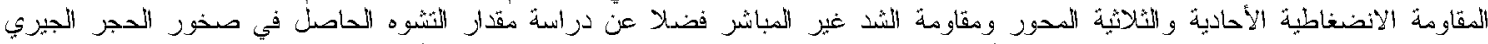

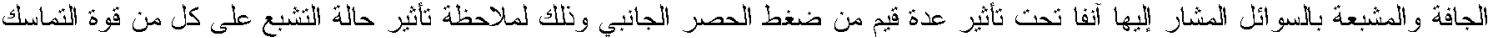
(Cohesion Force)

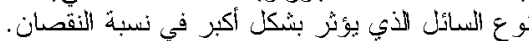

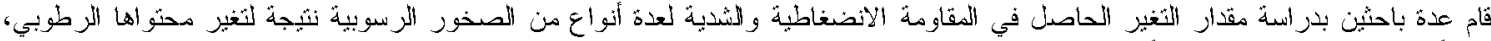

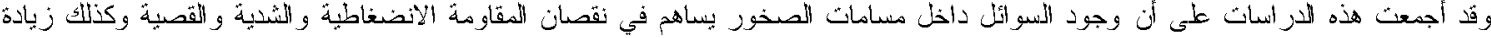

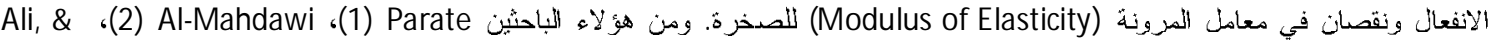

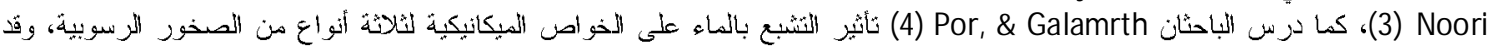

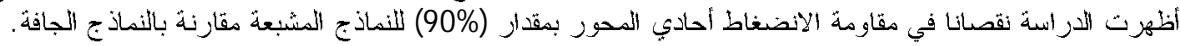

$$
\begin{aligned}
& \text { 2. المو اد المستخدمة في البحث } \\
& \text { 1.2 }
\end{aligned}
$$

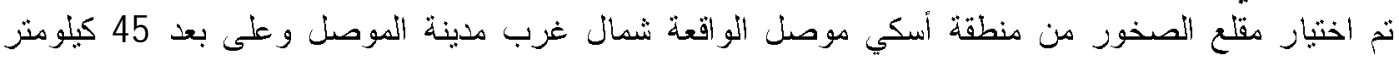

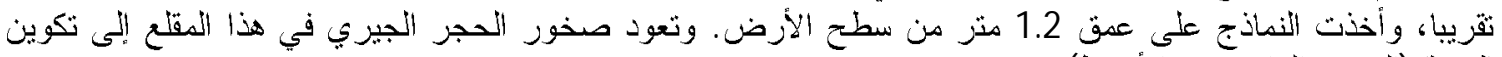

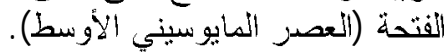

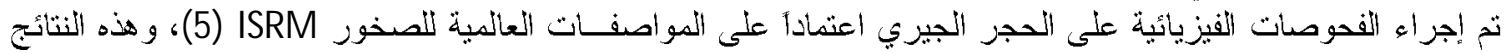
جذول (1) قيم بعض الخواص النفيزيائية للحجر الجيري.

\begin{tabular}{|c|c|c|}
\hline الانحر & معدل النتائج * & نوع الفحص \\
\hline & 2.06 & الكثافة الجافة (gm/cm³) \\
\hline & 2.66 & اللوزن النوعي (Gs) \\
\hline & 23.42 & المسامية المطلقة (n\%) \\
\hline
\end{tabular}
موضحة في الجنول (1).

تم فحص ستة نماذج من الصذّر لكل حالة.

كما أجريت فحوصات التحليل المعني للصخرة حيث أظهرت نتائج فحص الأشعة السينية الحائدة

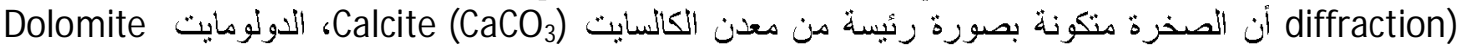

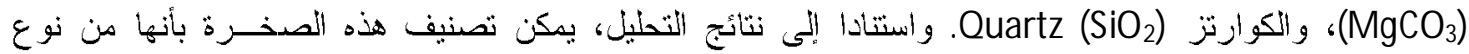
.(6) ASTM ،(Calcite Limestone)

2.2 السو ائل المستخدمة :

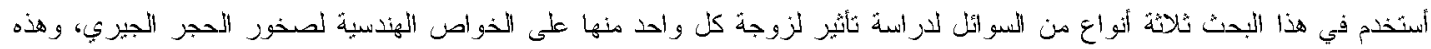

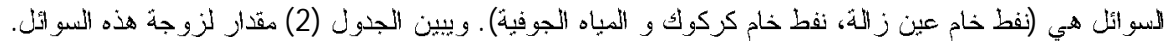


نوري : تأثيرنوعية السوائل على الخواص الهندسية لصخور الحجر الجيزي من منطقة أسكي موصل

$$
\text { الجنول (2) لزوجة النو ائل المستخدة في درجة حرارة } 22^{\circ} \text {. . }
$$

\begin{tabular}{|c|c|}
\hline اللزوجة ) & نوع النسائل \\
\hline 220 & نفط خام عين زالة \\
\hline 142 & نفط خام كركوك \\
\hline 8.85 & الكياه الجو فية \\
\hline
\end{tabular}

3. تحضير النماذج

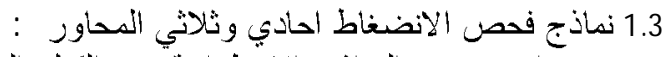

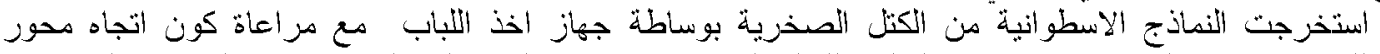

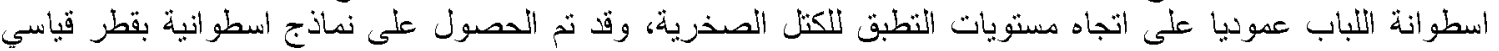

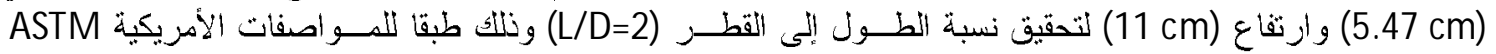

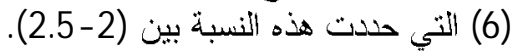

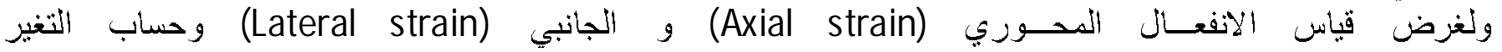

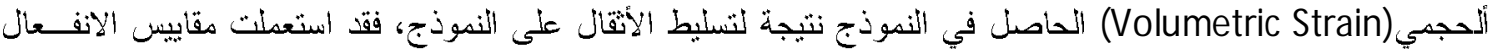

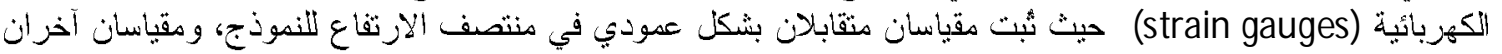
أفقيان منقابلان في منتصف الازتقاع ايضا وبموجب المواصفات العالمية الأمريكية ASTM (6) وكما موضح في الشكل

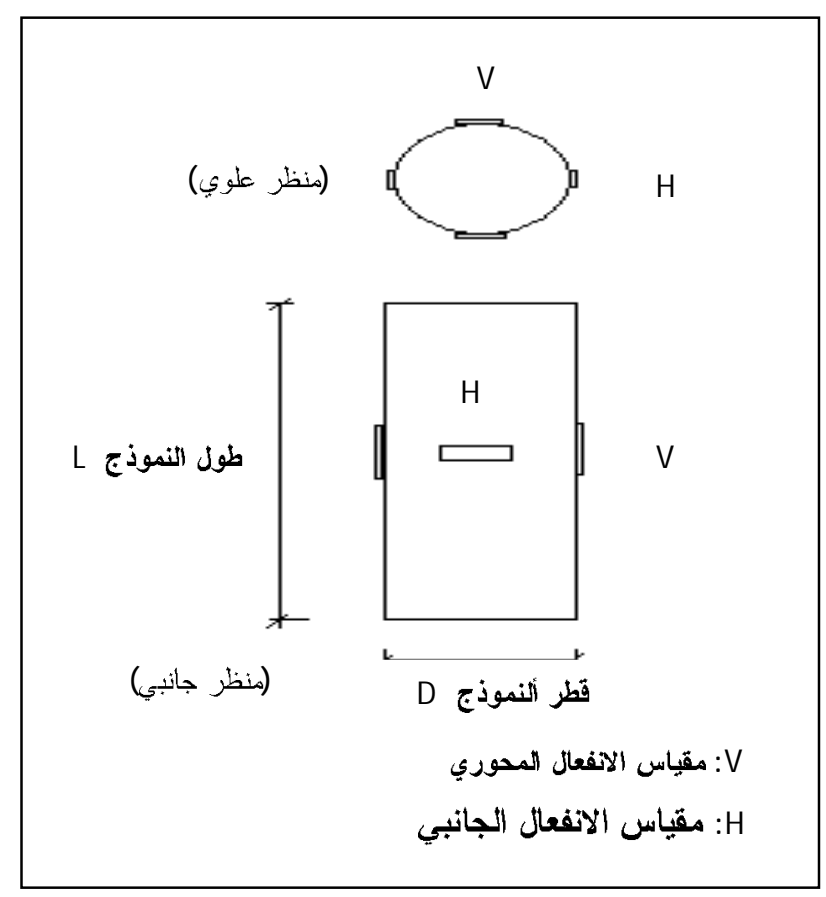

الشكل (1) مخطط توضيحي يبين أماكن لصق مقاييس الانفعال على النماذج الآسطو أنبة 


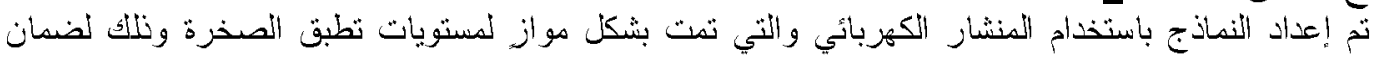

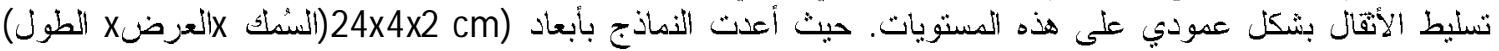

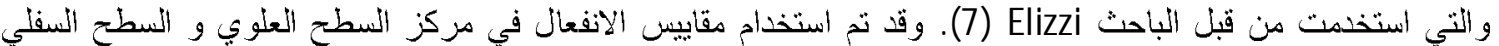

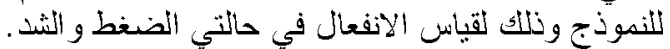
جفقت جميع النماذج بدرجة حرارة (C C (1050) لمدة 24 ساعة لنكون معدة للفحوصات لمطنوبة.

$$
\text { 4. عملية تشبيع النماذج }
$$

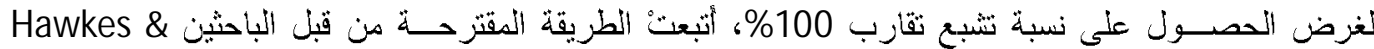

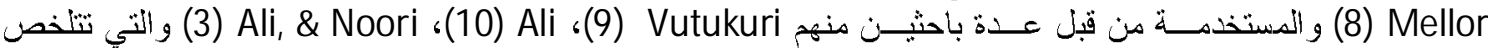

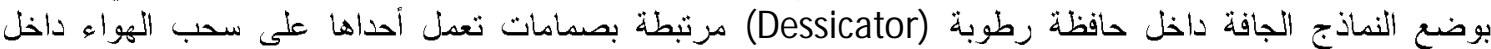

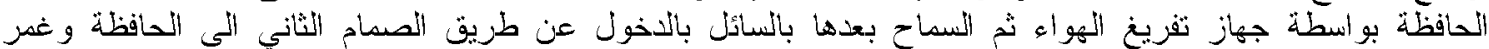

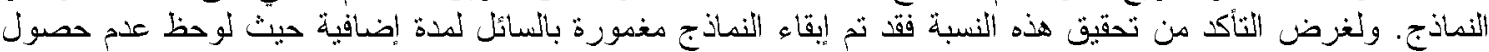

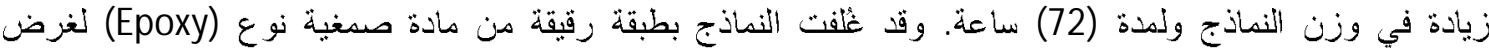

5. عملية قياس الانفعال

استخم في عطلية قياس الانفعال جهاز (strain-meter) و الكي يعمل بعد إيخال البيانات و الأوامر من خلال لوحة

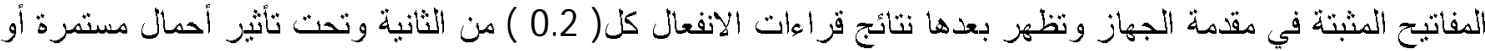
ثابتة. ويمكن ربط عدد من مقاييس الانفعال مع بعض بعها ليعطي الجهاز معدل نتائجها.

6. الفحوصات الميكانيكية تخ إجراء الفحوصات الميكانيكية التالية على صخور الحجز الجيري:

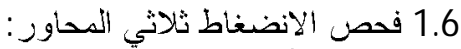

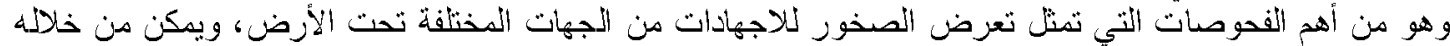

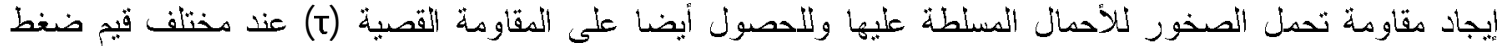

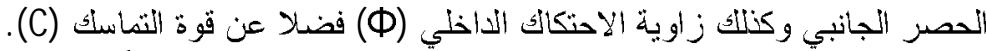

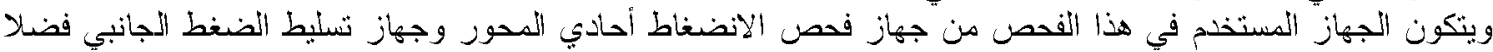

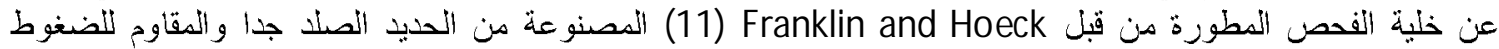

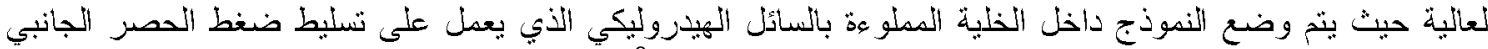

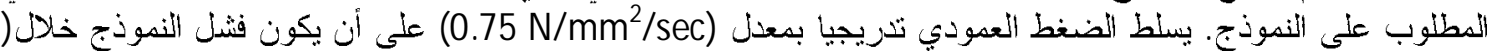

(6) ASTM دقيقة

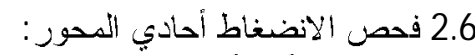
و هو أحد أنواع فحص الانضغاط ثلاثي المحاور إلا أن الضغط الجانبي يكون مساويا للصفر، و الجهاز المستخم

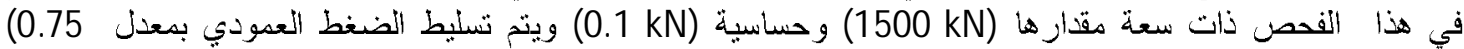

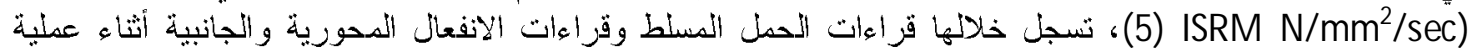

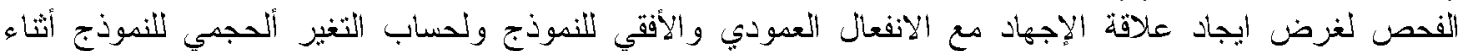

$$
\sigma_{c}=\frac{P}{A}
$$

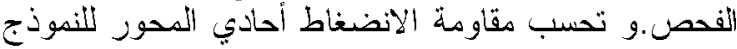




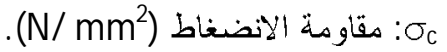

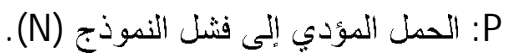

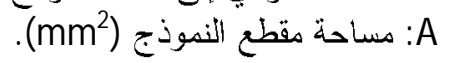

$$
3.6 \text { فحص الانحناء : }
$$

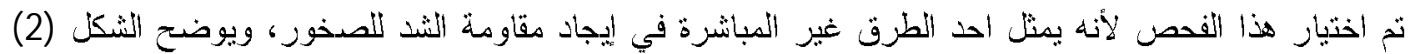

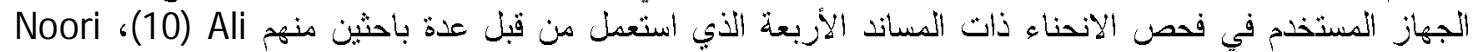

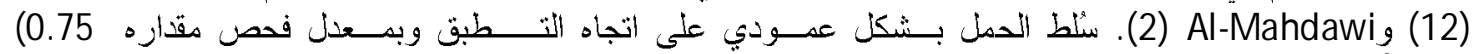

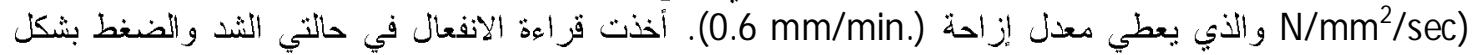

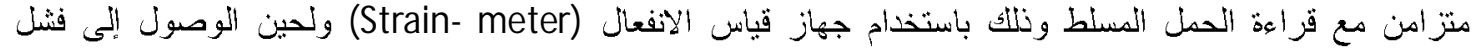

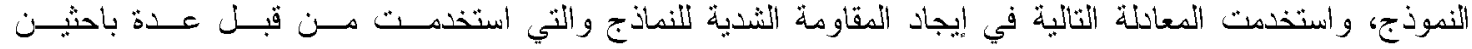

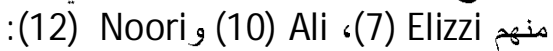

$\sigma_{t}=\frac{3 M\left(\varepsilon_{t}+\varepsilon_{c}\right)}{b d^{2} \varepsilon_{t}}$ إن أن:

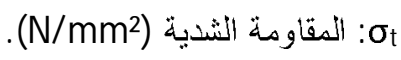
(

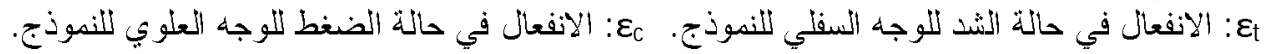

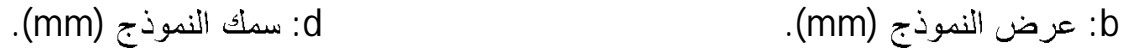

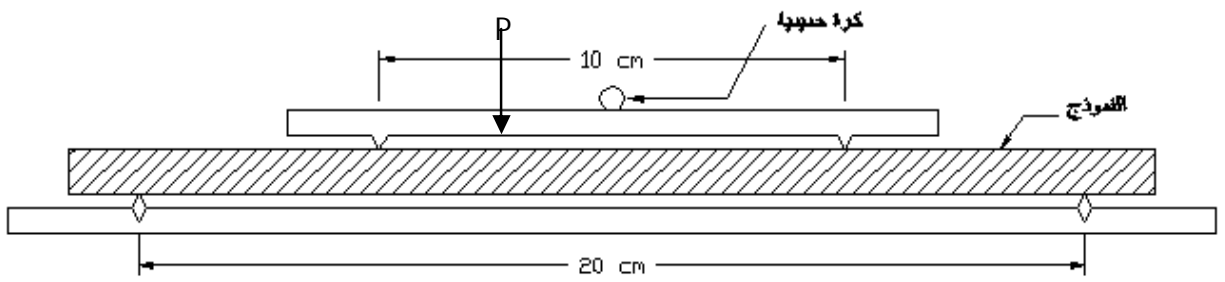

الشكل (2) المخطط التوضيحي للجهاز المستخدم في فحص الاحناء.

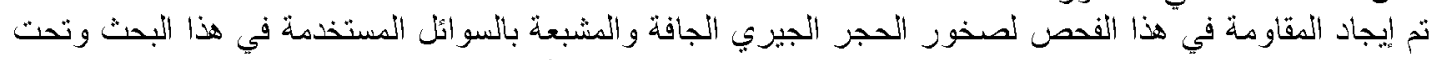

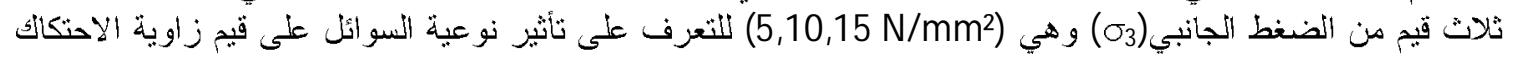

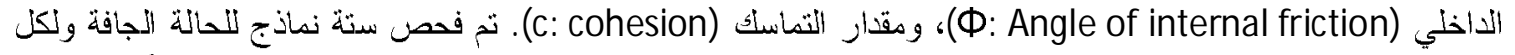

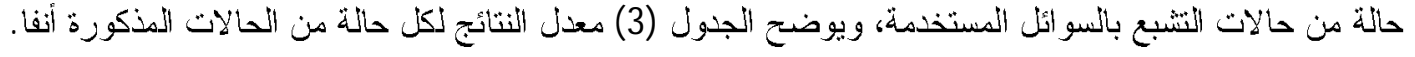


جذول (3) معدل نتائج فحص الانضغاط ثلاثي المحاور .

\begin{tabular}{|c|c|c|c|}
\hline 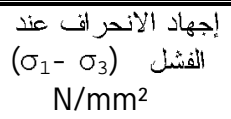 & الجنبي الحصر & الإجهاد الحصر ري N/mm² & حالة النشبع \\
\hline 23 & 5 & 28 & \multirow{3}{*}{ جاف } \\
\hline 31.7 & 10 & 41.7 & \\
\hline 40 & $\overline{15}$ & 55 & \\
\hline 19.75 & 5 & 24.75 & \multirow{3}{*}{ عنَ زالة } \\
\hline 27 & 10 & 37 & \\
\hline 34.35 & 15 & 49.35 & \\
\hline 17.15 & 5 & 22.15 & \multirow{3}{*}{ كزئ خام } \\
\hline 23.75 & 10 & 33.75 & \\
\hline 30 & 15 & 45 & \\
\hline 14.15 & 5 & 19.15 & \multirow{3}{*}{ المياه الجب فية } \\
\hline 19.39 & 10 & 29.39 & \\
\hline 24.65 & 15 & 39.65 & \\
\hline
\end{tabular}

يلاحظ من الجدول أن قيم إجهاد الاندر اف $\left(\sigma_{3}\right.$ أه

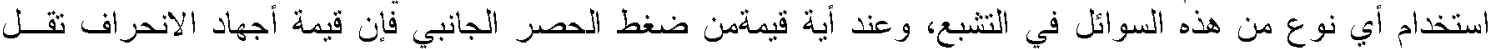

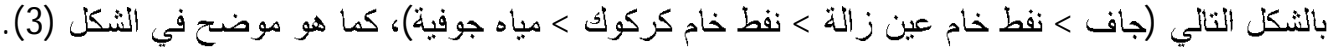

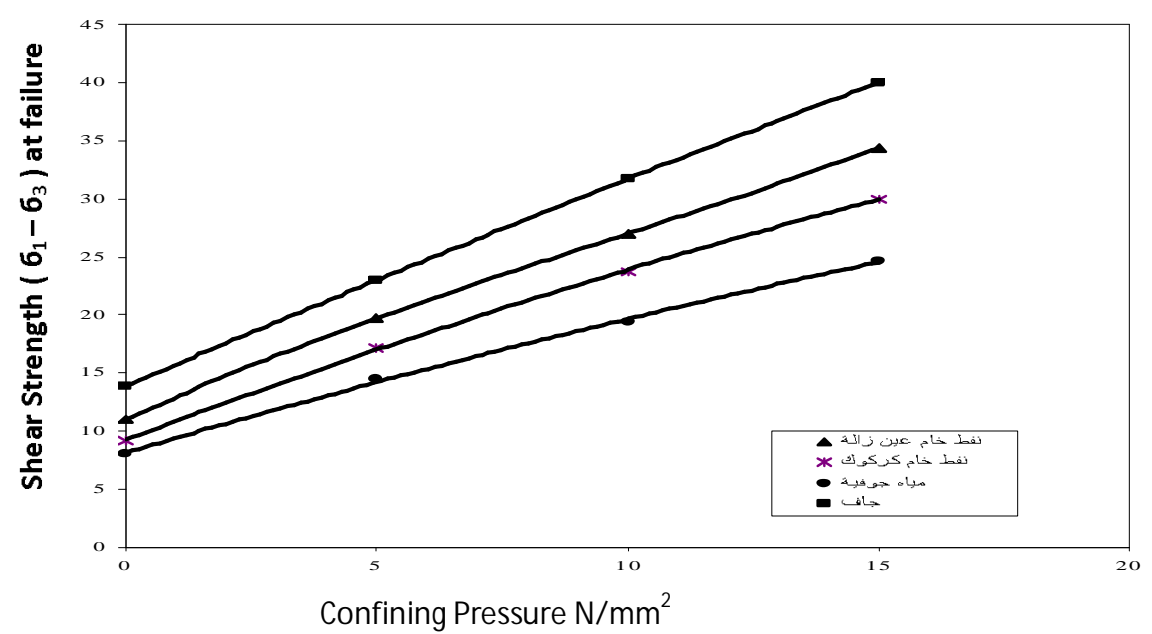

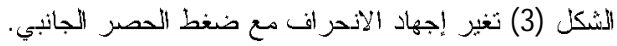

وقد لاحظ الباحثون Hart, \& wang (13)، Noori (14)، وكذلك (15) Lockner, \& Stanchists)، حصول

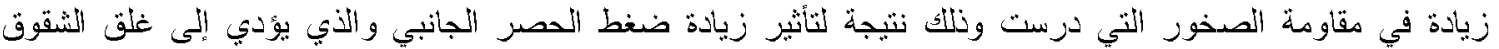
الثعزية وزيادة صدلابة هيكن الصذرة. كما وجد النباحث . Ballivy, et al (16) أن مقاومة الانضغاط ثلاثي الدحاور نتل

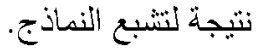

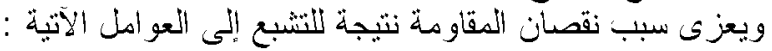

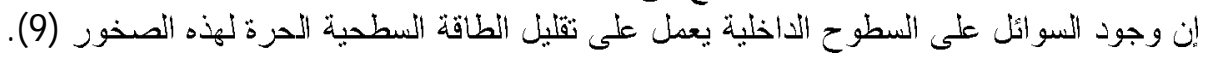




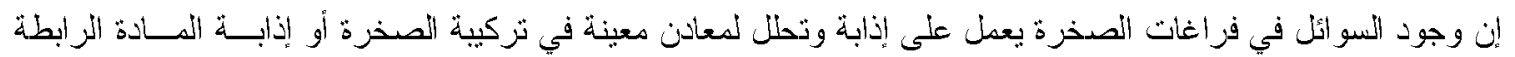

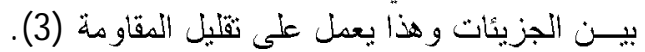

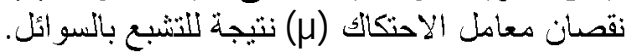

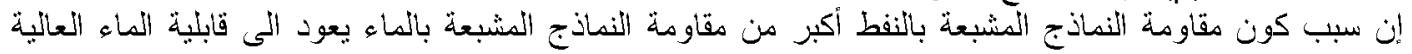

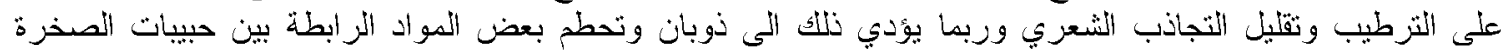

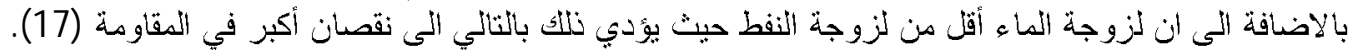

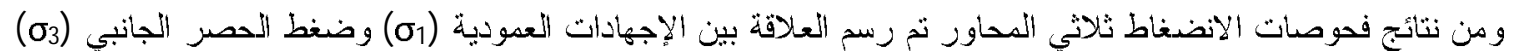

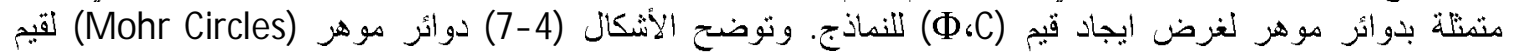

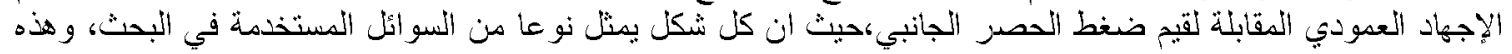

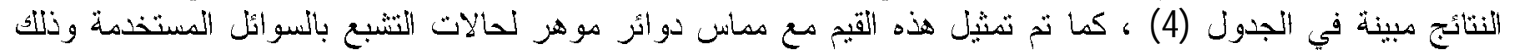

بالثكل (8).

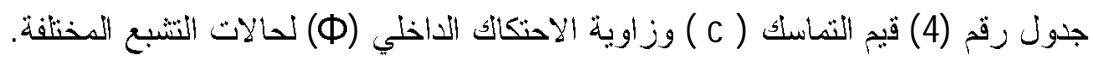

\begin{tabular}{|c|c|c|}
\hline 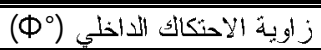 & النماسك (C/mm² (C) & حالْة التُشَبع \\
\hline 27 & 4.7 & جاف \\
\hline 25 & 4.11 & فقط خام عبِن ز اللة \\
\hline 23 & 3.44 & نفط خام كز كو كك \\
\hline 19.6 & 3.33 & المباه الجو فِبة \\
\hline
\end{tabular}

وييين الجدول (4) أن قيم التماسك (c) وزاوية الاحتكالك انداخلي (ه) تثل عن الحالة الجافة في حالة استخدام الأنواع التثلاثة

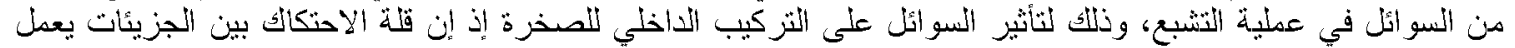

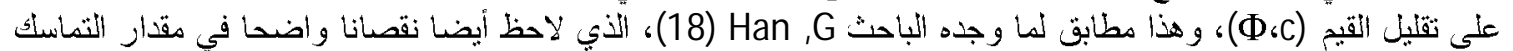

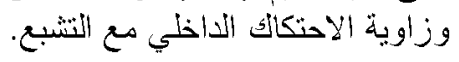

2.7 فحص الانضغاط أحادبي المحور :

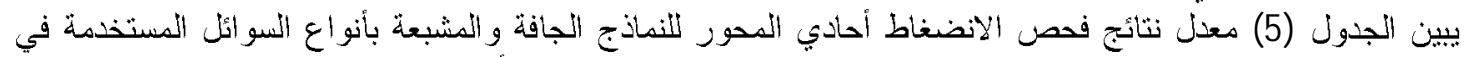

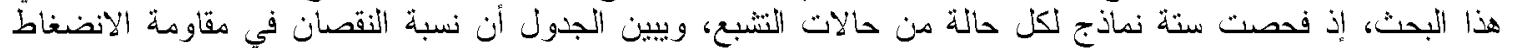

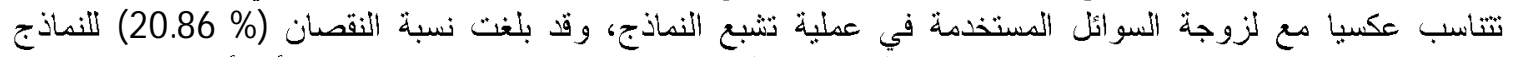

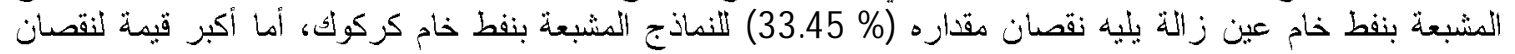

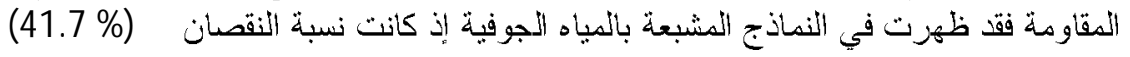

جدول(5)معنل نثائج فحص الانضغاط أحادي المحور للانماذج الجافةّ والمشبعة.

\begin{tabular}{|c|c|c|}
\hline 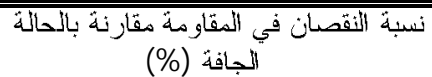 & 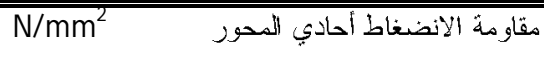 & حائة النشّبع \\
\hline & 13.9 & جان \\
\hline 20.86 & 11 & نفط خاد عبن ز اللة \\
\hline 33.45 & 9.25 & نفط خاد كر كر كا \\
\hline 41.7 & 8.1 & مياه جوفية \\
\hline
\end{tabular}




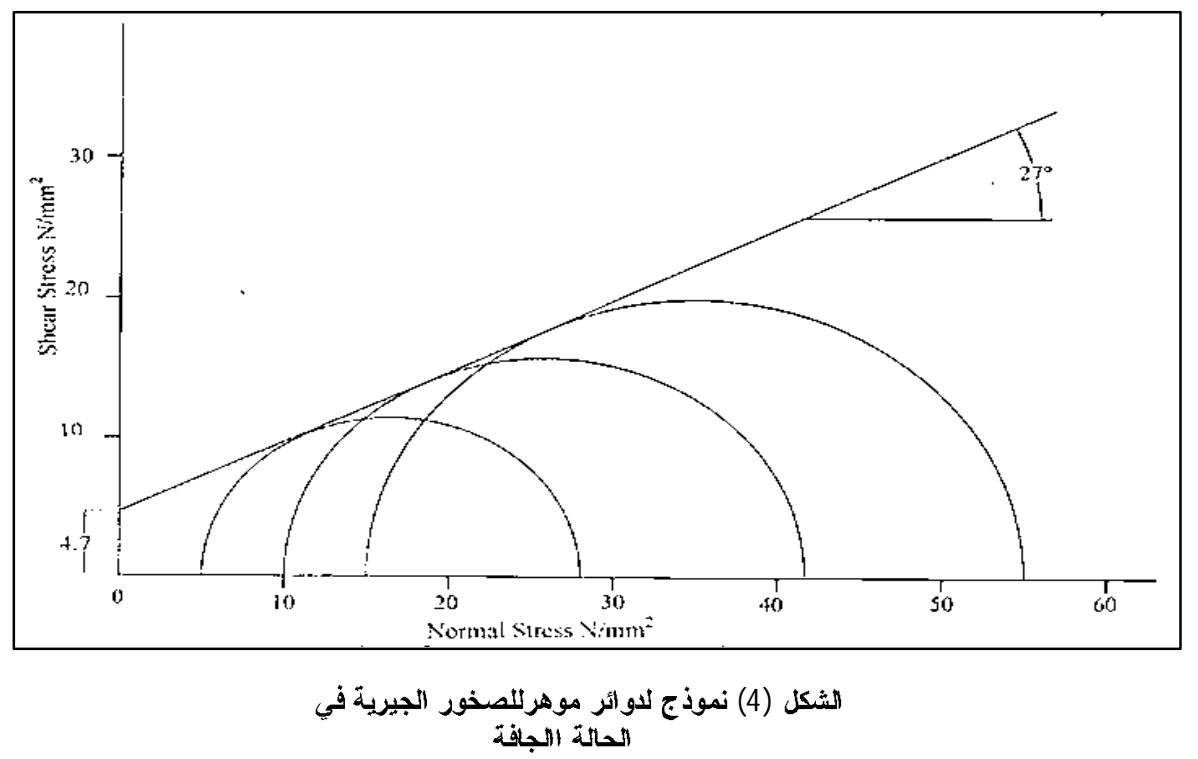

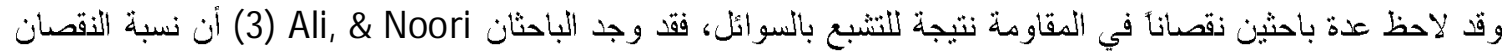

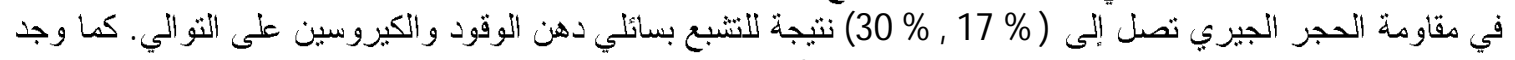

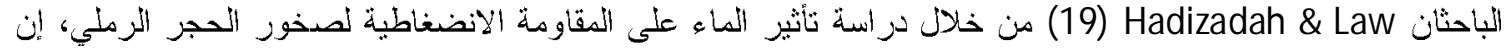
نسبة النقصان تصن إلى (45 (45) نتبجة لعملية التشبع بالماء. و يلاحظ أن الأسباب التي أدت إلى نقصان مقاومة الانضغاط أحادي المحور مشابهة للأسباب المذكوزة في الفقزة السابقة

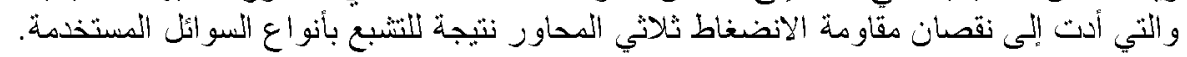

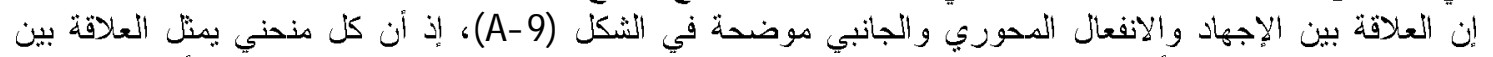

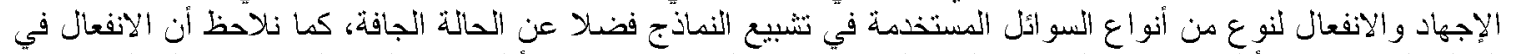

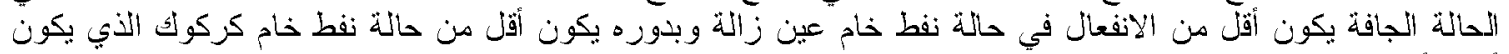

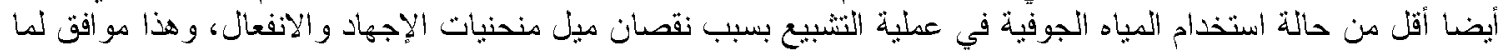

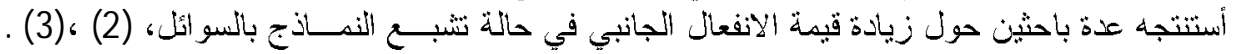

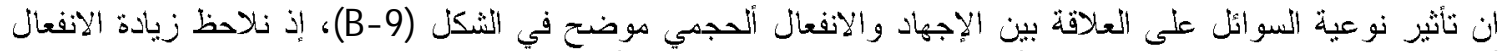

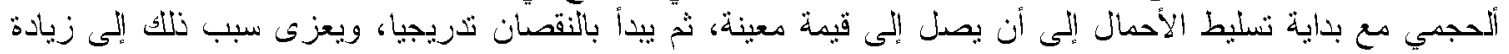

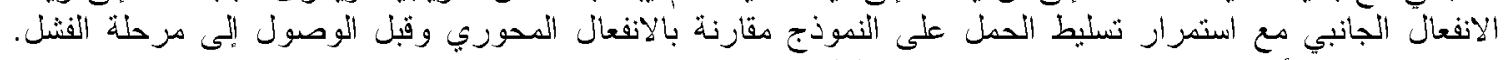

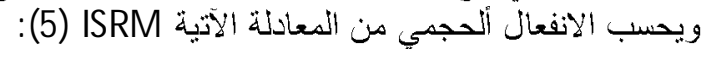

$$
\varepsilon_{v}=\varepsilon_{a}+2 \varepsilon_{l}
$$

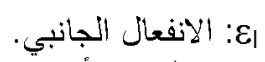

عa ع: الانفعال ألحجمي.

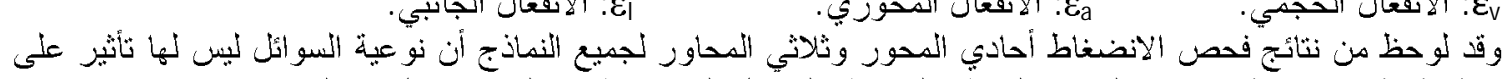

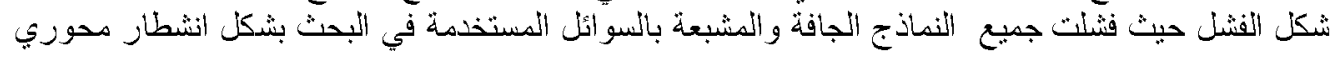




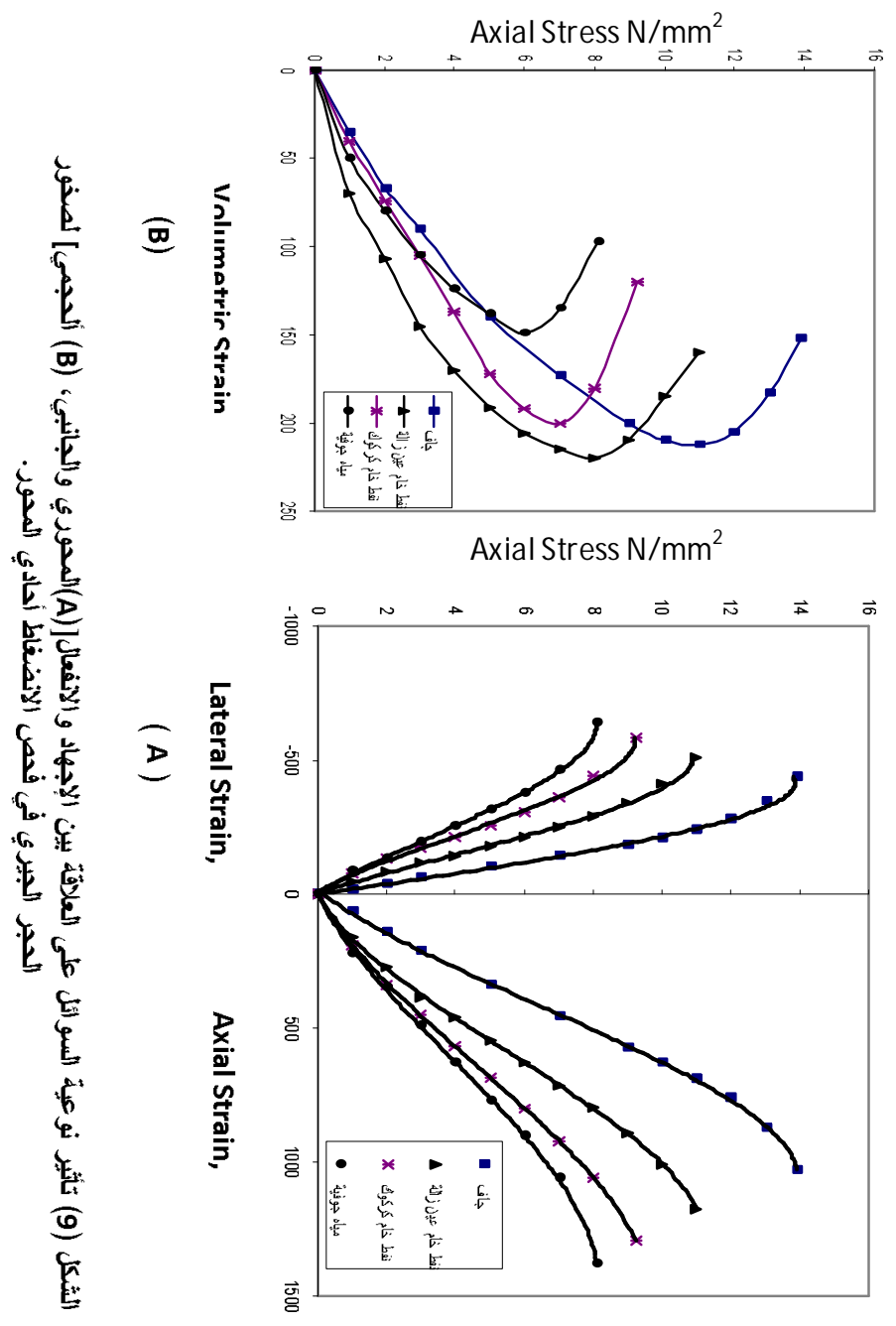

3.7 فحص الانحناء

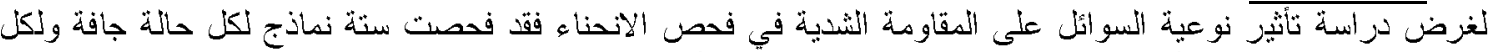

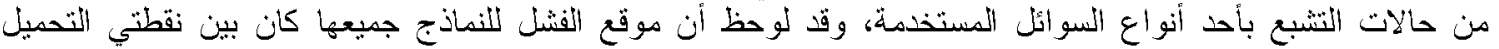

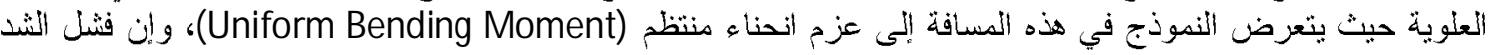

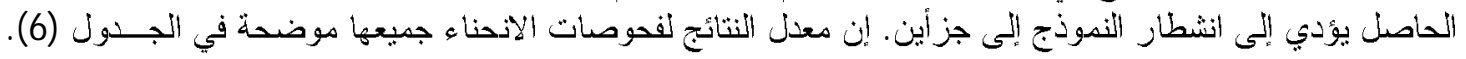

$$
\text { الجدول (6) معنل نتائج فحص الإنحناء للنماذج الجافة و المشبعة }
$$

\begin{tabular}{|c|c|c|}
\hline 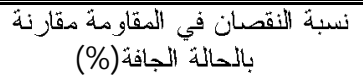 & المقاومة الشُدية (N/mm² & حالة التُشَبع \\
\hline$-\cdots$ & 4.61 & جاف \\
\hline 16.05 & 3.87 & نفط خام عبن ز اللة \\
\hline 24.08 & 3.5 & نفط خام كركوك \\
\hline 30.15 & 3.22 & البياه الجب فية \\
\hline
\end{tabular}




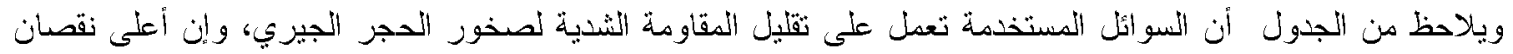

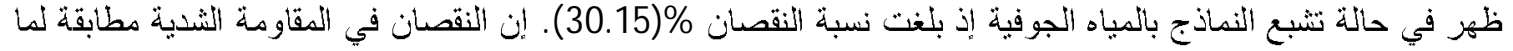

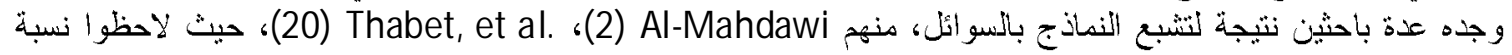

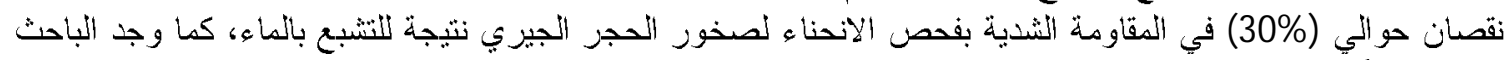

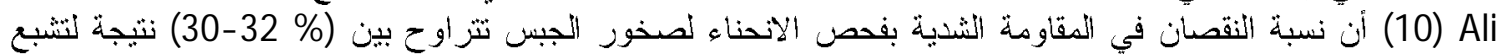

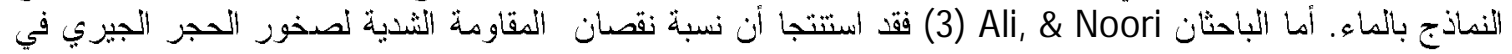

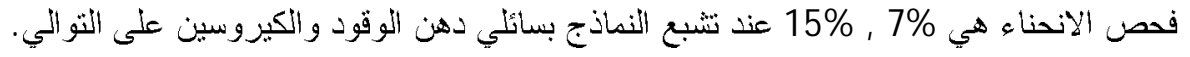

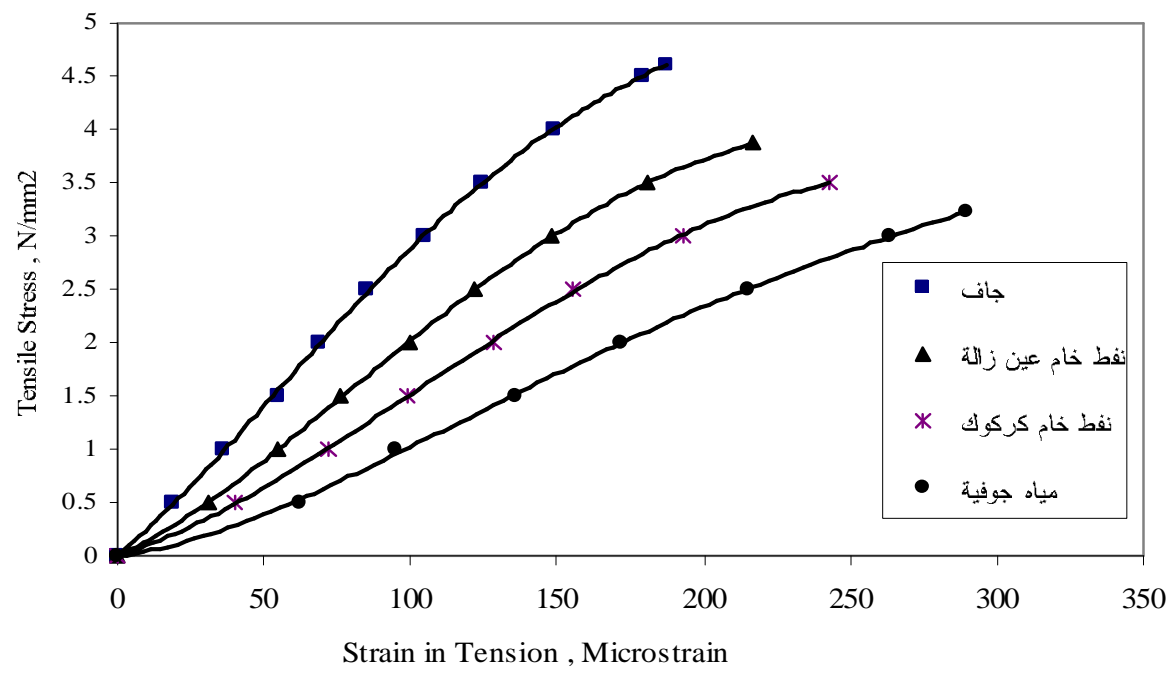

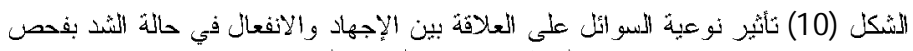

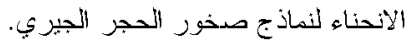

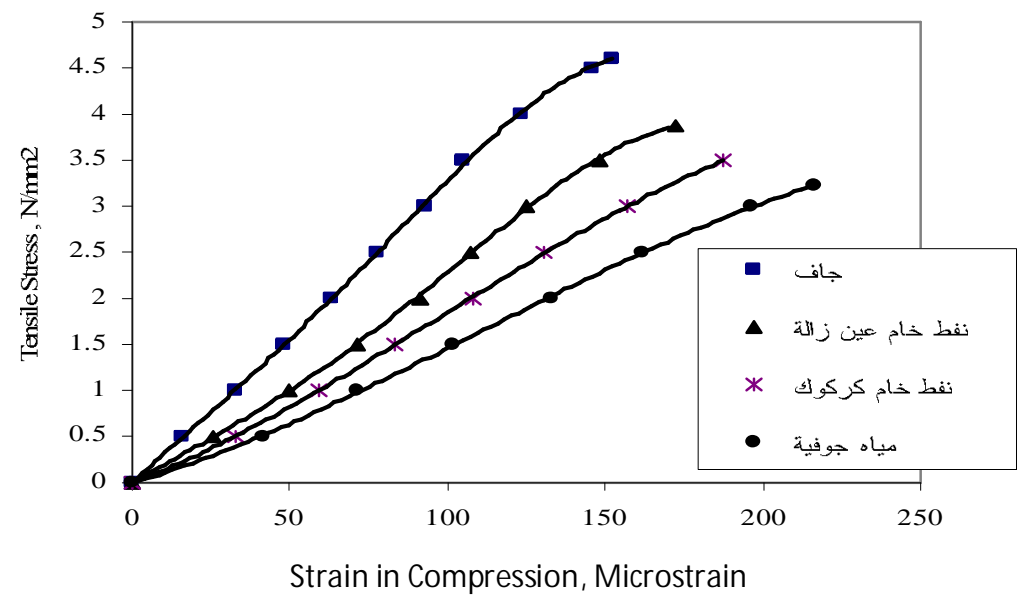

الشكل (11) ثأثثر نب عبة اللسو ائل على العلاقة بين الإجهات و الانفعان في حالة الضغط بفص 


$$
\text { نانتل الأشكال (10 -11)، العلاقة بين الإجهاد و الاتفعال في حالتي الثد و الضغط على التو الني في فحص }
$$

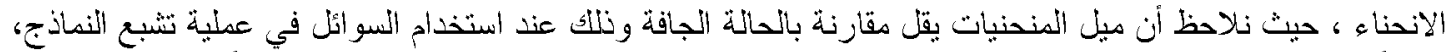

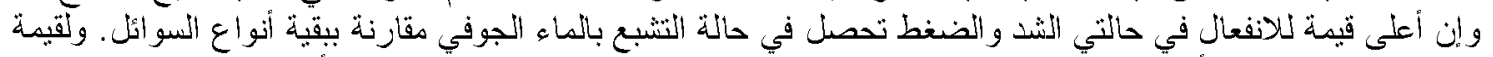

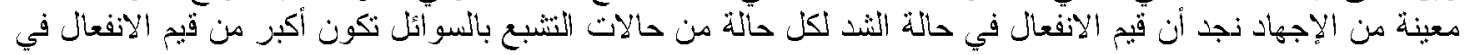

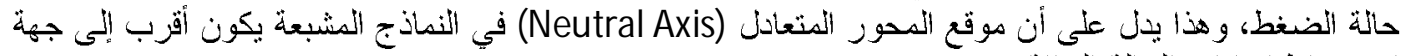

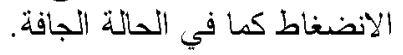

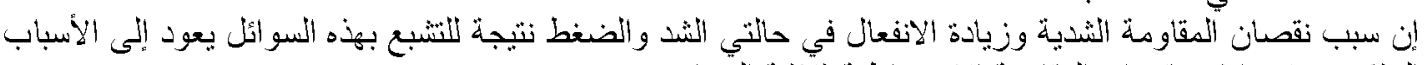

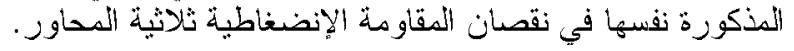

اعتمادا على نتائج الفحوصات التي أجريت في هذا البحث على صخور الحجر الجيزب في حالة تثبعها بالسو ائل (نفط خام

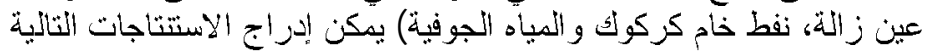

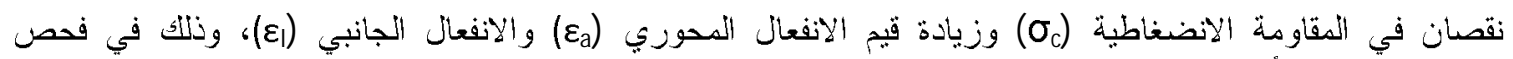

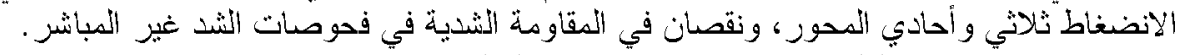

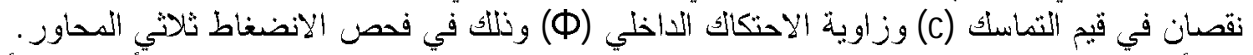

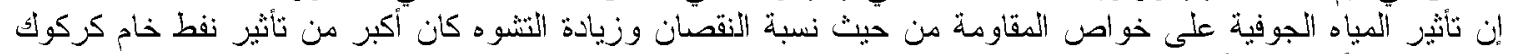

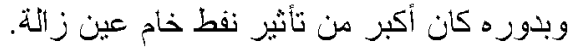

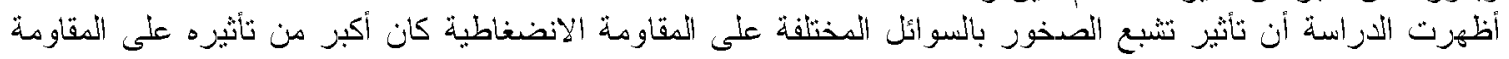
التشدية غيز المباندرة. إن شكل الفنشل لنماذج الصخور في الفحوصات جميعها لم يتأثز بنوع السائل المستخدم في عدلية التشبع.

Parate, N. S.," Influence of Water on The Strength of Limestone.", Transactions of AIME, Vol. 254 , PP 127131,(1973).

AL-Mahdawi, S.K., "Effect of Specimen Sizes and Water Saturation on Strength Properties of Jeribe Limestone", M.Sc. Thesis, civil Engg. Dept., College of Engineering, University of Mosul,(1985)..

Ali, S.A. \& Noori, T.M"Effect of Oils on The Strength and Deformation Properties of Limestone ", Proce. Of The Sixth Sci. Conference for Foundation Tech. Institutes. Baghdad - Iraq, pp. 226-232, (1998)..

Por, L. \& Galamrth, " Effect of Water Content on The Mechanical Behavior of Fine - Grained Sedimentary Rock ", Pizohishy Danishka, No.48,(2003).

ISRM., , "Suggested Methods for Determining Water Content, Porosity, Density, Absorption and Related Properties.", ISRM. Committee on Standardization of Laboratory Tests, Int. J. Rock Mech. Min. Min. Sci., Vol. 16 pp.143-156,(1979).

ASTM, Standards, " Soil and Rock "American Society for Testing and Material, Vol.04-08,(1989).

Elizzi, M.A, " time - Dependent Behavior of Some Evaporite Rocks ",Ph.D. Thesis, University of Sheffield, (1976). 
Hawkes, I. \& Mellor, M, "Uniaxial Testing in Rock Mechanics Laboratory ", Eng. Geol., Vol. 4, No. 3, pp.177-285, (1970).

Vutukuri, V.S., " The effect of the Liquids on the Tensile Strength of Limestone." Int. J. Rock Mech. Min. Sci. ,Vol. 11 pp. 27-29,(1974).

Ali, S.A., " Creep Properties of Evaporite Rocks with Particular Reference to Gypsum ", Ph.D. Thesis, University of Sheffield,(1979).

Franklin, J.A. \&Hoeck, E., "Developments in Triaxial Testing Technique" Rock Mechanics 2 , pp. 223-228,(1970).

Noori, T.m., " Study of The Long - Term Strength of Gypsum ", M.Sc. Thesis, civil Engg. Dept., College of Engineering, University of Mosul,(1989),

Hart, D.J., \& Wang, H.F., " Laboratory measurements of a complete set of poroelastic moduli for Berea sandstone and Indiana limestone ", J. Geophys. Res., No. 100, pp.741 - 751,(1995).

Noori, T.M., " Effect of Anisotropy on The Shear Strength Of Sandstone Rock in Triaxial Compression Test ", Raffidain Engineering Maga., No. 1 (1996),.

Lockner, D.A., \& Stanchits, S.A., " Undrained poroelastic response of sandstones to deviatoric stress change ", J. Geophys. Res., No. 107,(2002)

Ballivy, G., Ladan yi, B., \& Gill, D.E., " Effect of Water Saturation History on The Strength of Low - Porosity Rocks ", ASTM. STP 599, pp. 4 - 20,(1976).

Hellmann, R., Renders, P.J., Gratier, J., \&Guiguet, R"Experimental Pressure Solution Compaction of Chalk in Aqueous Solutions, Part 1. Deformation Behavior and Chemistry ", The Geochemical Society, Special Publication, No. 7, (2002) .

Han, G., " Rock Stability under Different Fluid Flow Conditions ", Ph.D., Thesis, chemical Engg. Dept., College of Engineering, University of Waterloo, Ontario, Canada,(2003).

Hadizadeh, J., \& Law. R.D., " Water-weakening of sandstone and quartzite deformed at various stress and strain rates ", Int. J. Rock Mech. Min. Sci. \& Geomech. Vol. 5, pp. 431 - 439,(1991).

Thabet, K.M., Khattab, S.I., \& Al - Azzo, S.I., , "Geotechnical Characteristics of Some Limestone in Nineveh, Iraq ", Confidential Report No. SM/SRC 10/1995,University of Mosul,(1995).

$$
\text { تم اجر اء البحث في كلية الهندسة - جامعة الموصل }
$$

